\title{
La política coreana de seguridad energética e implicaciones para la cooperación con América del Sur
}

\author{
Korean policy in the field of energy security: \\ implications for cooperation with South America
}

Won-Ho Kim*

\section{Resumen}

Como país inherentemente pobre en recursos naturales, Corea del Sur necesita explotarlos en los países en que existen en abundancia, siempre y cuando estos puedan asegurar un suministro estable. La política coreana pertinente apunta a establecer la estabilidad del suministro como «prioridad en la agenda nacional», reforzar la cooperación en recursos energéticos y minerales con países estratégicamente importantes, aumentar las inversiones en el desarrollo de estos recursos, y reforzar la base de apoyo para los sectores de negocios que persigan desarrollar recursos en el exterior. América Latina es una region importante para la política de seguridad energética de Corea del Sur. En el último tiempo la región ha sido el principal destino de la inversión coreana para la explotación de los recursos energéticos. El gobierno coreano también ha aplicado una activa estrategia de cooperación con sus socios de la región para este fin mediante comités bilaterales, y continuando su política de envío de delegaciones de alto nivel e incluso de visitas de Jefes de Estado. Sin embargo, la distancia y la falta de vínculos culturales seguirán sien-

Profesor, Graduate School of International Area Studies, Universidad de Hankuk, Corea. 
do barreras que habrá que superar para lograr que aumenten las inversiones coreanas en la región.

Palabras clave: energía, seguridad energética, noreste de Asia, Corea del Sur, Corea del Norte.

\section{Abstract}

As a country poorly endowed with natural resources, South Korea has no other option but to develop those resources abundant in other countries in order to secure their continuing supply. In this regard Korea's policy is oriented toward establishing a stable energy supply at the "top of the national agenda," strengthening energy and mineral resources cooperation with countries of strategic importance, expanding investment funds for the development of these resources, and strengthening the basis of support for the business sector in overseas resources development. Latin America is an important region for Korea's energy security policy. The region has recently been the main destination of Korean investments for energy resources development. The Korean government has also pursued an active strategy of cooperation with Latin American partners by means of bilateral committees, and the exchange of high-level delegations including heads of state. Nevertheless, distance and lack of cultural links will continue to be barriers preventing increased investments in the region.

Keywords: energy, energy security, Northeast Asia, South Korea, North Korea. 
Won-Ho KIM • La política coreana de seguridad energética...

\section{INTRODUCCIÓN}

Corea del Sur es un país pobre en recursos naturales debido a la limitada extensión de su territorio, y también a las características de este, al igual que Japón, Taiwán y la parte este de China. Históricamente, el sur de Corea ha tenido ventajas comparativas en agricultura mientras que el norte en minería. La división de la península en 1945 dificultó aún más el abastecimiento de recursos naturales en el país. Estas limitaciones en la dotación de recursos naturales incrementaron su dependencia de las importaciones de materias primas durante el dinámico proceso de industrialización iniciado en los años sesenta. Corea del Sur logró superar estas limitaciones emergiendo como centro de manufacturas en los años setenta y ochenta, y como centro de alta tecnología en los años noventa, logrando estabilizar sus cuentas comerciales. Aunque la economía coreana se transformó gradualmente en una basada en el conocimiento, su concentración en las industrias pesadas y petroquímicas y el estilo de vida de la sociedad coreana, crecientemente habituada al uso de vehículos, han convertido la seguridad energética en un tema importante a nivel nacional.

A pesar de que Corea del Sur debe asegurar la provisión estable de materias primas, la actual inestabilidad de los precios internacionales representa un gran desafío para el gobierno surcoreano, el sector industrial, y los hogares. Además, se prevé un aumento del in- tercambio intercoreano en los próximos años, lo que a mediano y largo plazos puede dar lugar a una amplia integración económica y política. En el marco de la Negociación de Seis Partes sobre el controversial programa nuclear de Corea del Norte, en julio 2005 Corea del Sur ofreció proporcionarle ayuda energética en forma de dos gigawatts de electricidad a partir de 2008, mediante la construcción de nuevas plantas eléctricas y la ampliación de la red del Sur para aliviar la fuerte escasez de energía en el Norte. Como Corea del Norte no ha invertido en el desarrollo de recursos naturales tales como antracita, hidroelectricidad y carbón, tiene una enorme demanda de energía, que aumentará mucho cuando su economía se abra a la sociedad internacional. En consecuencia, Corea del Sur necesitará desarrollar un sistema consolidado de provisión energética en preparación para una futura unificación (Ryu, 2005). En fin, en esta coyuntura de cambio a nivel internacional y regional Corea del Sur enfrenta un múltiple desafío económico, estratégico y de seguridad nacional. En consecuencia, como país inherentemente pobre en recursos naturales, necesita explotar recursos naturales en países en que ellos existan en abundancia, siempre y cuando ellos puedan asegurarle un suministro estable.

Este trabajo intenta explorar el desarrollo de las políticas coreanas de recursos naturales en el exterior y analizar las implicaciones de estas políticas para la colaboración con América La- 
tina. En la sección siguiente se ofrecerá un panorama general del incremento de los precios de las materias primas $\mathrm{y}$ sus efectos en el proceso de toma de decisiones. Se incluye debido a que el trabajo procura revisar las estrategias coreanas de búsqueda de recursos en el contexto del elevado precio actual de las materias primas. Luego, se analiza el grado de dependencia coreana de recursos naturales como antecedente para el proceso de elaboración de políticas, y se dan a conocer las actuales estrategias de explotación de recursos naturales en el exterior. A continuación, se examina la situación de las reservas, la producción y el consumo en América Latina y el Caribe, para luego revisar el presente estatus de la relación de Corea del Sur con la región en temas relacionados con la seguridad energética. Para terminar, se examina la agenda política y sus implicaciones para las inversiones del país en sectores extractivos en América Latina.

\section{LAS ALZAS RECIENTES DEL PRECIO DE LAS MATERIAS PRIMAS}

Mientras que el aumento de los precios de las materias primas es una buena noticia para los países exportadores, como Corea del Sur, no lo es para países que dependen de su importación. Las alzas de precios constituyen un desafío para los países exportadores en cuanto a la manera de manejar sus crecientes ingresos por concepto de exportaciones (Gottschalk y Patres, 2006), y para los países importadores en cuanto a cómo manejar el impacto que producen estas alzas y soportarlas a largo plazo. Los precios de la energía también han fluctuado, afectando fuertemente la planificación de las políticas de desarrollo económico de Corea del Sur. Esto quedó demostrado cuando a comienzos de 1980 el país sufrió la primera recesión desde el milagro económico; las recientes crisis internacionales de energía también han provocado gran inquietud.

Las fluctuaciones mencionadas son producto de los cambios tanto en el mercado de demanda como en las coyunturas políticas internacionales. Varios factores explican la tendencia al alza de los precios de las materias primas y permiten pronosticar que han de mantenerse.

Primero, la variable más importante de la reciente alza de los precios de las materias primas es la demanda de petróleo, minerales y productos agrícolas provocada por la rápida industrialización china, el llamado "Chinaboom", que se acompaña de la demanda de otros países asiáticos, como la India. Incluso se sostiene que el tamaño e índice de crecimiento de China y la India pueden conducir a un cambio estructural mayor en los precios relativos, que a largo plazo dará lugar a una tendencia al aumento del comercio de materias primas. Se observa que los precios reales de las materias primas se mantienen por debajo del promedio registrado entre 1960 y 1985 (Gottschalk y Prates, 2006: 2-9, 25). 
En gran medida, el aumento sostenido de los precios del petróleo se atribuye al fuerte crecimiento de las economías asiáticas de uso intensivo de petróleo. En 2004, el 14\% del incremento total de la demanda de petróleo correspondió a China e India, lo que significó el $3.5 \%$ del total, comparado con el $1 \%$ en 2002 (IMF, 2005). Se proyecta que en los próximos 20 años el consumo chino de petróleo aumentará de 5.56 millones de barriles por día a 12.8 millones de barriles diarios. Por lo tanto, la mayor cantidad de petróleo deberá ser importada (NYT, 1 de marzo de 2005).

Segundo, la reactivación de las economías de los países desarrollados ha sido un importante componente del crecimiento global de la demanda de materias primas.

Tercero, las incertidumbres relacionadas con la guerra de Irak, las epidemias como el SARS o la gripe aviar, la significativa influencia política de la OPEP, la intensificación de la competencia por gas natural limpio, entre otras temas internacionales, desanimaron la inversión en infraestructura (sistemas de almacenamiento y transporte) por los sectores productores de materias primas, pese a las señales de reactivación de la economía global.

Como el mundo enfrenta una creciente crisis energética, Corea del Sur debe formular e implementar medidas políticas con el objeto de establecer un sistema de seguridad sostenible y de largo plazo.

\section{LA DEPENDENCIA COREANA DE RECURSOS}

Debido a la estructura industrial de uso intensivo de energía, a la insuficiencia de recursos a nivel interno y a la dependencia de la importación de recursos naturales, para Corea del Sur es fundamental asegurarse el abastecimiento de energía y minerales en el extranjero.

Para que el país logre la seguridad energética tiene varias opciones: no solamente petróleo sino también carbón, gas natural y energía nuclear. El uso de carbón disminuyó drásticamente en los años ochenta debido a sus efectos negativos en el medio ambiente y la salud humana, y a su baja rentabilidad. Se cerraron numerosas minas de carbón del país. La energía nuclear surgió como principal alternativa durante el primer shock del petróleo, y en 1987 su participación en la generación eléctrica alcanzó su punto máximo al superar el $50 \%$, para caer al $37.8 \%$ en 2003 , y a $36.0 \%$ en 2004 . No obstante, Corea ocupa el sexto lugar del mundo en generación eléctrica nuclear, con 124 mil millones de kwh en 2004, después de Estados Unidos (789 mmkwh), Francia (426 mmkwh), Japón (272 mmkwh), Alemania (159 mmkwh), y Rusia (137 mmkwh) (DOE, 2006). Sin embargo, la energía nuclear ya no parece ser una alternativa sostenible para la comunidad local, que a partir de los años noventa se ha manifestado cada vez más contraria al impacto ambiental producido por las plantas nucleares 
y ha exigido la reducción gradual o el cierre de los reactores. Por otro lado, Corea del Sur está en condiciones relativamente menos favorables porque carece de gasoductos internacionales para el gas natural y lo importa en forma de LNG (gas natural licuado). Por la falta de incentivos a la investigación, la energía alternativa nunca ha sido una prioridad nacional. Todo esto hace que el país dependa del petróleo en un
$47.0 \%$ del consumo de energía primaria, mientras que el promedio mundial es de $36.4 \%$. Le siguen el carbón con $24.4 \%$, la energía nuclear con $14.8 \%$, el gas natural con $13.3 \%$ y la hidroelectricidad con $0.5 \%$ (BP 2006).

La tabla que figura a continuación muestra el grado de dependencia del petróleo y de recursos minerales importados de Corea del Sur en 2005.

TABLA I

DEPENDENCIA DE RECURSOS IMPORTADOS (2005)

\begin{tabular}{|l|c|c|c|}
\hline Materia prima & Consumo & Importación & Tasa de dependencia \\
\hline Petróleo (millones BbI) & 761.4 & 843 & $100 \%$ \\
\hline Gas Natural (millones T) & 23.1 & 22.3 & $100 \%$ \\
\hline Carbón bituminuoso (millones T) & 69.3 & 69.3 & $100 \%$ \\
\hline Uranio (UT) & 2.747 & 3.776 & $100 \%$ \\
\hline Cobre(1000T) & 976 & 925 & $100 \%$ \\
\hline Hierro (millón T) & 44.1 & 43.5 & $99 \%$ \\
\hline Zinc(1000T) & 761 & 766 & $100 \%$ \\
\hline Tierra (T) & 6.981 & 7.398 & $100 \%$ \\
\hline
\end{tabular}

Fuente: MOCIE, 2006a.

En 2002, Corea del Sur dependió altamente de la importación de energía $(97.3 \%$ ), que representó el $20.7 \%$ del total de sus importaciones. Actualmente ocupa el cuarto lugar en el ranking internacional de importación de petróleo y el segundo en la importación de LNG. Se prevé que en 2020 la demanda total de recursos energéticos alcanzará a unos 340.3 millones TOE (tonelaje del equivalente del petróleo), esto es, 1.6 veces más que en 2002.

Además, el 79.4\% del crudo importado proviene de Medio Oriente, y el $55 \%$ del gas natural también viene de la región. La concentración en el Me- dio Oriente se desarrolló en los años noventa debido al bajo costo a corto plazo y a las avanzadas relaciones bilaterales entre Corea del Sur y los países de la región, caracterizadas por inversiones mutuas. Por un lado, esta modalidad de cooperación podrá servir a la seguridad energética relacionando los intereses privados de cada parte, especialmente con las inversiones de Dubai International, y firmas de Arabia Saudita y Abu Dhabi en el puerto de contenedores de Busan y en las compañías petroleras de Corea del Sur (Calder, 2005); por el otro, esto demuestra que el país necesita diversificar las fuentes 
de suministro de recursos naturales en el exterior debido a la vulnerabilidad del Medio Oriente a conflictos internacionales (KEEI, 2004a).

\section{El Desarrollo Coreano DE RECURSOS EN EL EXTERIOR}

Corea del Sur comenzó a desarrollar proyectos petroleros en el exterior en 1981, cuando la empresa coreana Codeco Energy puso en marcha el «Proyecto Madura Occidente» en Indonesia. Hacia finales del año 2002 el país había emprendido un total de 109 proyectos en 37 naciones. Sin embargo, más de la mitad de ellos terminaron por la falta de rentabilidad. En 2005, Corea del Sur llevaba a cabo 65 proyectos de desarrollo de petróleo y gas en 26 países.

Hacia fines de diciembre de 2002, compañías privadas habían invertido 2.741 millones de dólares y la Corporación Nacional Coreana de Petróleo unos 1.224 millones de dólares en este tipo de proyectos, esto es, un total de 3.965 millones de dólares. Estos proyectos recibieron ayuda de recursos de inversión, préstamos para energía y cuentas para proyectos especiales por unos 860 millones de dólares, equivalentes al $21.7 \%$ del total. Los propios recursos de la Corporación Nacional Coreana de Petróleo alcanzaron a 2.255 millones de dólares o $56.9 \%$ del total de las inversiones nacionales (KEEI, 2004b).

El siguiente gráfico muestra la composición de los 65 proyectos de petróleo y gas:

Entre ellos, en América Latina se

\section{GRÁFICO I}

\section{Proyectos de Petróleo y gas}

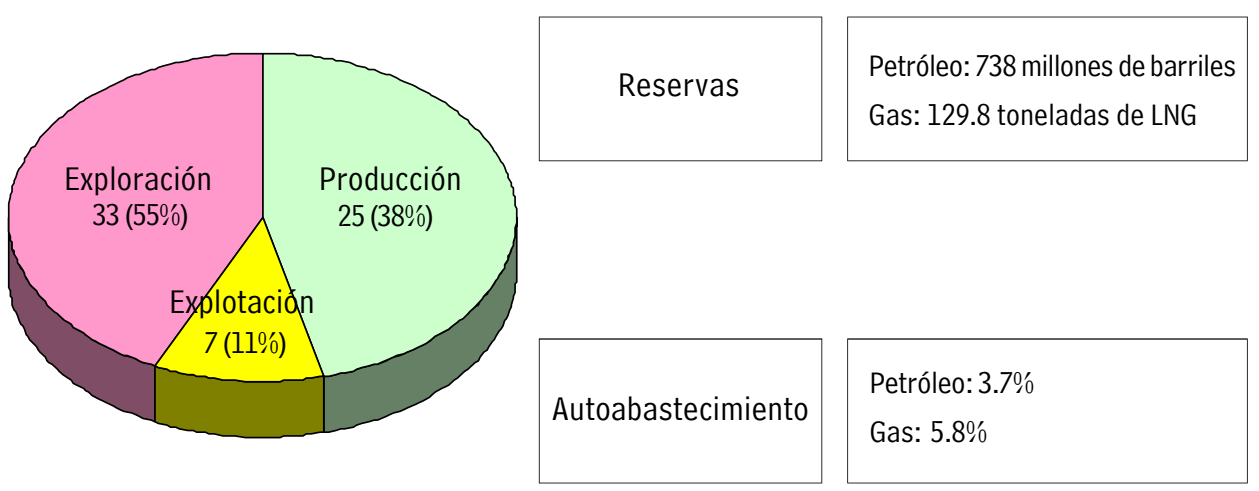

Fuente: MOCIE, 2006a. 
realizan 13 proyectos en 5 países, como se detalla en la Tabla 2: $15 \%$ del total en exploración, $29 \%$ en explotación, y $24 \%$ en producción. Con la inversión de 206 millones de dólares (22\%), América Latina se sitúa detrás de Asia y Australia con 410 millones de dólares $(43 \%)$. Le siguen el Medio Oriente con 114 millones de dólares (12\%), África con 102 millones de dólares (11\%), Rusia y Europa con 90 millones de dólares (9\%), Asia Central con
17 millones de dólares $(2 \%)$ y América del Norte con 14 millones de dólares $(1 \%)$.

Otros 114 proyectos tienen por objeto el aprovechamiento de recursos de carbón y minerales en 29 países. En total, hay 179 proyectos que apuntan a desarrollar recursos naturales en 44 países. El siguiente gráfico muestra la composición de los 114 proyectos de carbón y minerales.

GRÁfICO 2

Proyectos de CARbón y MiNerales

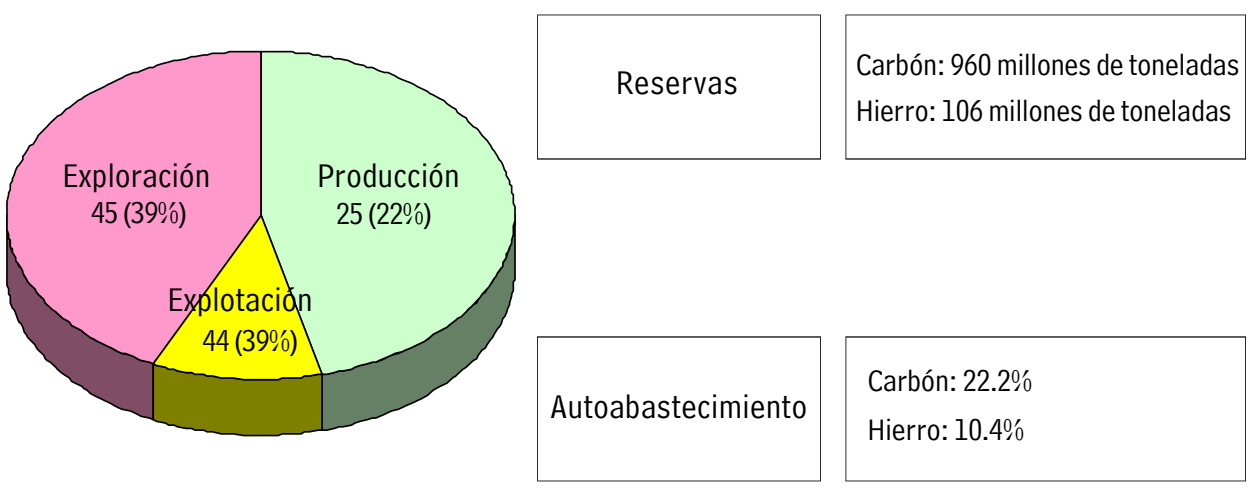

Fuente: MOCIE, 2006a.

\section{LA ORIENTACIÓN DE LA POLÍTICA DE SEGURIDAD ENERGÉTICA}

Corea del Sur tiende a ser un importante inversionista en países en vía de desarrollo, fundamentalmente debido a sus estrategias de exploración de recursos y comercialización. Mientras tanto, los elevados precios actuales de las materias primas exigen aumentar la IED en el exterior, que se destina a sectores de recursos naturales.
Al respecto, la política coreana apunta lo siguiente (MOCIE, 2006a):

- Establecer que la estabilidad del suministro es "prioridad en la agenda nacional»

- Reforzar la cooperación en recursos energéticos y minerales con países estratégicamente importantes

- Aumentar las inversiones en el desarrollo de recursos energéticos y minerales

- Reforzar la base de apoyo para los 
sectores de negocios que busquen desarrollar recursos en el exterior.

Primero, de acuerdo con la política orientada a establecer el suministro de energía como prioridad de la agenda nacional, el gobierno coreano estableció que es el Comité Nacional de Energía, presidido por el presidente de la república. El Comité surgió a raíz de la aprobación por la Asamblea Nacional de la Ley Básica de Energía en febrero de 2006, y se reunió por primera vez el 28 de noviembre de 2006. Una de sus principales funciones es revisar las medidas de apoyo al desarrollo de recursos en el exterior a nivel gubernamental. En febrero de 2001, el gobierno coreano había preparado el Primer Plan Básico para el Desarrollo de Recursos en el Exterior (2001-2010), y en diciembre de 2004 completó el Segundo Plan Básico (2004-2013). La idea del gobierno era elaborar y poner en práctica un plan básico decenal cada tres años para asegurar el suministro estable de energía y recursos minerales, vitales para el desarrollo económico. La primera reunión del Comité Nacional de Energía produjo el informe Visión de la Energía 2030, que plantea la meta de "autoabastecimiento energético», o dependencia de energía explotada con capitales nacionales, en $18 \%$ de la demanda local en el año 2013, y en 35\% en 2030, a contar del $4.1 \%$ en 2005 (IHT, 2006; MOCIE, 2006d).

Segundo, de acuerdo con la política de reforzar la cooperación con socios estratégicos en materia de recursos, en los últimos años el gobierno coreano ha perseguido activamente la llamada «diplomacia de recursos» mediante conversaciones del presidente de la nación con sus contrapartes de países ricos. Para combinar estratégicamente capitales y tecnología coreanos con energía y recursos naturales de estas se ha promovido el «Esquema de cooperación Ganador-Ganador». Como consecuencia de ello, el Comité Bilateral en Cooperación de Recursos Energéticos y Minerales ha suscrito acuerdos con Brasil, México, Uzbekistán, y otros países. Su objetivo principal es diversificar la cooperación energética. Los mayores socios coreanos en cuestiones de energía producen energía en Medio Oriente y el sudeste de Asia, en circunstancias que el Medio Oriente representa el $79 \%$ de sus importaciones de energía, Asia el 12\%, África el 4\%, y América el $3.7 \%$. Sin embargo, como la competencia para asegurar las fuentes de energía se intensificará en el futuro, Corea del Sur tiene que diversificar su estructura de abastecimiento energético, incluyendo países del noreste de Asia, Asia Central, África y América Latina.

En particular, en el noreste de Asia, China y Japón ya están empeñados en una fuerte competencia para asegurar el abastecimiento de energía. Sus esfuerzos al respecto han incluido negociaciones con Rusia y ofrecimientos de proveer a Moscú de ayuda financiera para instalar oleoductos en territorios rusos. Es cierto que la demanda de energía en el noreste de Asia, que incluye Corea del Sur, China, Japón, y Taiwán, 
ha producido un crecimiento económico mundial dinámico que tiende a ser abruptamente ascendente. Esta situación producirá una feroz competencia entre esos países para asegurarse el abastecimiento de recursos energéticos.

En este contexto, Rusia ha surgido como fuente clave de este abastecimiento en el noreste asiático. El extremo oriente de Rusia es conocido especialmente por sus enormes reservas de petróleo y gas natural, así como por sus recursos hídricos, que pueden utilizarse para generar energía hidráulica. Además, Rusia ha estado tratando de suministrar petróleo y gas natural de sus yacimientos de Siberia oriental a Corea del Sur, China, y Japón. El autoabastecimiento de energía en la región podría aumentarse considerablemente si se establecieran redes de transporte eficientes. Esto contribuiría mucho a establecer bases sólidas para el suministro eficiente de energía y para aumentar la seguridad energética regional. Asimismo, la seguridad energética coreana se vería considerablemente impulsada si se importaran recursos energéticos desde zonas cercanas. Frente al ya sostenido aumento de la demanda de gas natural en el futuro, a Corea del Sur le interesa desarrollar una estructura de suministro estable a largo plazo en cooperación con Rusia y China, lo que implicaría esfuerzos coordinados para importar el gas natural de gasoductos en las áreas de Siberia oriental así como de Irkutsk y Sakhalin. Al mismo tiempo, Corea del Sur podría importar electricidad desde Rusia mediante la co- nexión de líneas de transmisión rusas con las redes de suministro del norte y sur de Corea. La instalación de tuberías y electricidad contempla redes en conjunción con proyectos de cooperación energética con la participación de Corea del Norte, que bien podrian conducir a establecer un sistema de suministro de energía intercoreano integrado, asegurando el abastecimiento de energía para ambas Coreas (Ryu 2005). En este sentido, el proyecto de ductos en el noreste asiático involucra a la vez cooperación y competencia entre países vecinos de la región para la seguridad energética de cada uno de ellos.

Sin embargo, para los inversionistas coreanos Rusia no parece tan confiable como las oportunidades que ofrece. La política interna y externa en torno a los recursos petroleros rusos ha sido una de las frecuentes polémicas contemporáneas. Cuando Rusia advirtió en octubre de 2006 que suspendería el proyecto de petróleo y gas Sakhalin-2 dirigido por la Royal Dutch, por valor de 22 mil millones de dólares, a la Korea Gas, que había celebrado un contrato para importar gas por 20 años a partir de abril de 2008 le produjo gran inquietud. Esto es solo un ejemplo que indica que Corea del Sur debe buscar alternativas en todo el mundo. Al respecto, rápidamente parecen atractivos Asia Central y África que están relativamente menos dominados por las principales compañías petroleras (IHT, 2006). Aun así, hay factores de riesgo político respecto de la cooperación con Rusia y con Corea del Norte. 
Won-Ho KIM • La política coreana de seguridad energética...

Tercero, de acuerdo con la política dirigida a la expansión de los recursos de inversión para el desarrollo de la energía y los recursos naturales, el gobierno coreano ha ampliado el financiamiento gubernamental a empresas de extracción mediante la reciente introducción de incentivos fiscales, incluyendo rebajas impositivas y descuentos de las tarifas de contribución especial. Además, el gobierno ha incrementado su capacidad de inversión y financiamiento para el desarrollo de recursos en el exterior mediante la ampliación de los Fondos Especiales de Energía y elevando el máximo del fondo estratégico del Banco Coreano de Exportación e Importación (KEXIM). También se ha estudiado la posibilidad de establecer un Fondo de Garantía de Deudas. Sin embargo, el financiamiento que otorga el gobierno de Corea del Sur es superior al de otros países. En efecto, en 2007 los recursos de inversión dispuestos para estos proyectos, incluyendo los recursos de apoyo especiales, se elevaron a 470 millones de dólares y el total del fondo de inversión acumulativa fue de 4 mil millones, lo cual equivale a la décima parte de lo que otorga su contraparte japonesa. Además, sostuvo por sí solo el $41 \%$ del fondo de inversión BP, que en 2002 representó unos 9.7 mil millones de dólares (KEEI, 2004b). También es interesante observar que:

[Las empresas chinas] pueden distribuir la ayuda del gobierno para asegurar contratos, ... y utilizar líneas fuertes de crédito de las instituciones financieras del gobierno chino ... [Ellas] tien- den a hacer ofertas poco rentables, usar préstamos bilaterales y financiación china, y gastar desordenadamente... los inversionistas chinos persiguen objetivos de mercado y estratégicos, más bien que comerciales (NYT, 1 de marzo de 2005).

Durante cinco años, de 2001 a 2006 , las empresas petroleras chinas invirtieron 15 mil millones de dólares en la compra de yacimientos petrolíferos y compañías petroleras en unos 100 países. Por su parte, India invirtió $5 \mathrm{mil}$ millones de dólares en energía en 14 países (IHT, 2006). El gobierno coreano había seguido aumentando su presupuesto de recursos de inversión para el desarrollo de energía y recursos naturales, y finalmente realizó un incremento mayor hasta alcanzar un total de 1 billón 602 mil millones de won (1.12 mil millones de dólares) en 2007, a partir de 664.1 mil millones de won (706.4 millones de dólares), en 2006, lo que significó un $60 \%$ de aumento (MOCIE, 2006e). Esa suma incluye la inversión en recursos internos e internacionales. Esta importante alza simboliza la percepción coyuntural del gobierno sobre la crisis energética y al mismo tiempo la amenaza que constituye la intensa competencia internacional en materia de seguridad energética.

Finalmente, para reforzar la infraestructura y apoyar el sector de negocios que busca desarrollar recursos en el extranjero, el gobierno coreano ha puesto en marcha programas para fomentar la creación de firmas especializadas en este tipo de actividades, me- 
jorar la preparación de especialistas en tecnología y capacitar profesionales en el desarrollo de recursos minerales, además de facilitar la participación del sector privado en proyectos atractivos y estimular la percepción pública acerca de la importancia del desarrollo de los recursos minerales.

\section{LOS SECTORES EXTRACTIVOS EN} AMÉRICA LATINA Y LAS INVERSIONES COREANAS

Como lo revelan las estadísticas, América Latina y el Caribe es una región en que abundan los recursos energéticos. En efecto, cuenta con el $9.8 \%$ de las reservas del mundo y con $13.2 \%$ de la producción total, y ocupa el tercer lugar después del Medio Oriente y Europa/Eurasia. Además, junto con el Medio Oriente y África, es exportador neto de petróleo, dado que su producción sobrepasa el consumo. Entre otros países, se destaca Venezuela, miembro de la OPEP, que cuenta con el $68 \%$ de las reservas de la región. En materia de producción, México, Venezuela y Brasil representan $79.2 \%$ del total regional.

En cambio, América Latina y el Caribe no se destaca en materia de reservas y producción de gas natural, y

TABLA 2

RESERVAS, PRODUCCIÓN Y CONSUMO DE PETRÓleO, POR REGIONES (2005)

\begin{tabular}{|l|c|c|c|c|c|c|}
\hline & \multicolumn{2}{|c|}{$\begin{array}{c}\text { Reservas } \\
\text { (miles de millones de } \\
\text { barriles) }\end{array}$} & \multicolumn{2}{|c|}{$\begin{array}{c}\text { Producción } \\
\text { (mil bl/d) }\end{array}$} & \multicolumn{2}{|c|}{$\begin{array}{c}\text { Consumo } \\
\text { (mil bl/d) }\end{array}$} \\
\cline { 2 - 7 } & & Participación & & Participación & & Participación \\
\hline $\begin{array}{l}\text { América del } \\
\text { Norte }\end{array}$ & 45.8 & $3.8 \%$ & 9,877 & $12.2 \%$ & 22,897 & $27.8 \%$ \\
\hline $\begin{array}{l}\text { América } \\
\text { Latina y el } \\
\text { Caribe }\end{array}$ & 117.2 & $9.8 \%$ & 10,713 & $13.2 \%$ & 6,754 & $8.2 \%$ \\
\hline $\begin{array}{l}\text { Europa/ } \\
\text { Eurasia }\end{array}$ & 140.5 & $11.7 \%$ & 17,534 & $21.6 \%$ & 20,350 & $24.7 \%$ \\
\hline $\begin{array}{l}\text { Medio } \\
\text { Oriente }\end{array}$ & 114.3 & $61.9 \%$ & 25,119 & $31.0 \%$ & 5,739 & $7.0 \%$ \\
\hline África & 742.7 & $9.5 \%$ & 9,835 & $12.1 \%$ & 2,763 & $3.4 \%$ \\
\hline $\begin{array}{l}\text { Asia } \\
\text { Pacífico }\end{array}$ & 114.3 & $3.3 \%$ & 8,000 & $9.9 \%$ & 23,957 & $29.1 \%$ \\
\hline Mundo & $1,200.7$ & $100.0 \%$ & 81,078 & $100.0 \%$ & 82,460 & $100.0 \%$ \\
\hline
\end{tabular}

Fuente: BP 2006.

Incluye México en América Latina y el Caribe. 
Won-Ho KIM • La política coreana de seguridad energética...

TABLA 3

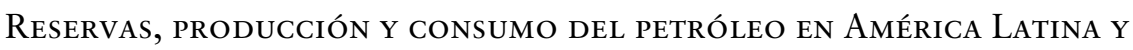
el CARIBE (2005)

\begin{tabular}{|l|c|c|c|c|}
\hline & $\begin{array}{c}\text { Reservas (miles } \\
\text { de millones de } \\
\text { barriles) }\end{array}$ & $\begin{array}{c}\text { Producción(A) } \\
\text { (mil bl/d) }\end{array}$ & $\begin{array}{c}\text { Consumo(B) } \\
\text { (mil bl/d) }\end{array}$ & $\begin{array}{c}\text { A-B } \\
(\mathrm{mil} \mathrm{b} / \mathrm{d})\end{array}$ \\
\hline México & 13.7 & 3,759 & 1,978 & 1,781 \\
\hline Argentina & 2.3 & 725 & 421 & 304 \\
\hline Brasil & 11.8 & 1,718 & 1,819 & -101 \\
\hline Colombia & 1.5 & 549 & 257 & 292 \\
\hline Ecuador & 5.1 & 541 & 230 & 311 \\
\hline Perú & 1.1 & 111 & 148 & -37 \\
\hline Trinidad yTobago & 0.8 & 171 & & 2,454 \\
\hline Venezuela & 79.7 & 3,007 & 553 & $-1,066$ \\
\hline Otros & 1.3 & 142 & 1,208 & 3,959 \\
\hline $\begin{array}{l}\text { América Latina y } \\
\text { el Caribe }\end{array}$ & 117.2 & 10,713 & 6,754 & \\
\hline
\end{tabular}

Fuente: BP 2006.

solo cuenta con el $4.1 \%$ del total mundial. Venezuela ocupa el primer lugar en reservas, mientras que en la producción de gas natural se destacan Argentina y México. Trinidad y Tabago, Bolivia, y Argentina exportan gas natural. De acuerdo con su programa extractivo Venezuela, Perú, y Colombia tienen potencial para ser exportadores netos (Ver Tablas 4 y 5 en la página siguiente).

La situación en materia de reservas y exportación de petróleo o gas natural no explica las decisiones de inversión extranjera en sectores extractivos. En muchos casos, la política de explotación de los recursos naturales es más importante que las reservas. Entre los países preferidos por el capital extranjero, en 1997 Brasil abrió sectores upstream (búsqueda, explotación y producción) a las empresas extranjeras al promulgar la Ley de Petróleo $N^{\circ}$ 9478, 1997; en
2003, el gobierno de Luiz Inacio Lula da Silva hizo lo mismo con sectores downstream (refinación, transporte y venta). Mientras que unas 40 compañías extranjeras participan en sectores upstream de Brasil, la empresa estatal Petrobrás prácticamente domina los sectores downstream. Al modificar su legislación en 2003, Colombia promueve y facilita inversiones extranjeras extractivas para no convertirse en importador de petróleo. Unas 30 compañías extranjeras participan en el desarrollo de yacimientos colombianos. La Constitución del Perú garantiza el tratamiento de la inversión extranjera en pie de igualdad con la nacional, y en 1997 abolió el monopolio de la energía por la empresa estatal Petróleos del Perú.

En cambio, un creciente número de países latinoamericanos monopolizan o nacionalizan los recursos naturales. Pese a que Petróleos de México 
TABLA 4

RESERVAS, PRODUCCIÓN Y CONSUMO DEL GAS NATURAL POR REGIÓN (2005)

\begin{tabular}{|c|c|c|c|c|c|c|}
\hline & \multicolumn{2}{|c|}{$\begin{array}{c}\text { Reservas } \\
\text { (billones de } \mathrm{m}^{3} \text { ) }\end{array}$} & \multicolumn{2}{|c|}{$\begin{array}{c}\text { Producción } \\
\text { (miles de millones de } \\
\mathrm{m}^{3} \text { ) }\end{array}$} & \multicolumn{2}{|c|}{$\begin{array}{c}\text { Consumo } \\
\text { (miles de millones de } \\
\mathrm{m}^{3} \text { ) }\end{array}$} \\
\hline & & Participación & & Participación & & Participación \\
\hline $\begin{array}{l}\text { América del } \\
\text { Norte }\end{array}$ & 7.05 & $3.9 \%$ & 711.1 & $25.7 \%$ & 724.9 & $26.4 \%$ \\
\hline $\begin{array}{l}\text { América } \\
\text { Latina y el } \\
\text { Caribe }\end{array}$ & 7.43 & $4.1 \%$ & 175.1 & $6.34 \%$ & 173.7 & $6.32 \%$ \\
\hline $\begin{array}{l}\text { Europa/ } \\
\text { Eurasia }\end{array}$ & 64.01 & $35.6 \%$ & $1,061.1$ & $38.4 \%$ & $1,121.9$ & $40.8 \%$ \\
\hline $\begin{array}{l}\text { Medio } \\
\text { Oriente }\end{array}$ & 72.13 & $40.1 \%$ & 292.5 & $10.6 \%$ & 251.0 & $9.1 \%$ \\
\hline África & 14.39 & $8.0 \%$ & 163.0 & $5.9 \%$ & 71.2 & $2.6 \%$ \\
\hline $\begin{array}{l}\text { Asia } \\
\text { Pacífico }\end{array}$ & 14.84 & $8.3 \%$ & 360.1 & $13.0 \%$ & 406.9 & $14.8 \%$ \\
\hline Mundo & 179.85 & $100.0 \%$ & $2,762.9$ & $100.0 \%$ & $2,749.6$ & $100.0 \%$ \\
\hline
\end{tabular}

Fuente: BP 2006.

* Incluye México en América Latina y el Caribe.

TABLA 5

Reservas, Producción y CONSUmo del gas natural en América Latina y el CARIBe (2005)

\begin{tabular}{|c|c|c|c|c|}
\hline & $\begin{array}{c}\text { Reservas } \\
\text { (billones de } \mathrm{m}^{3} \text { ) }\end{array}$ & $\begin{array}{c}\text { Producción(A) } \\
\text { (miles de millones } \\
\text { de } \mathrm{m}^{3} \text { ) }\end{array}$ & $\begin{array}{c}\text { Consumo(B) } \\
\text { (miles de millones } \\
\text { de } \mathrm{m}^{3} \text { ) }\end{array}$ & $\begin{array}{c}\text { A-B } \\
\text { (miles de } \\
\text { millones de } \mathrm{m}^{3} \text { ) }\end{array}$ \\
\hline México & 0.41 & 39.5 & 49.6 & -10.1 \\
\hline Argentina & 0.50 & 45.6 & 40.6 & 5.0 \\
\hline Bolivia & 0.74 & 10.4 & - & 10.4 \\
\hline Brasil & 0.31 & 11.4 & 20.2 & -8.8 \\
\hline Chile & - & - & 7.6 & -7.6 \\
\hline Colombia & 0.11 & 6.8 & 6.8 & 0.0 \\
\hline Perú & 0.33 & - & 1.6 & -1.6 \\
\hline Trinidad y Tabago & 0.55 & 29.0 & - & 29.0 \\
\hline Venezuela & 4.32 & 28.9 & 29 & 0.0 \\
\hline Otros & 0.17 & 3.5 & 18.3 & -14.8 \\
\hline $\begin{array}{l}\text { América Latina y } \\
\text { el Caribe }\end{array}$ & 7.44 & 175.1 & 173.6 & 1.5 \\
\hline
\end{tabular}

Fuente: BP 2006. 
Won-Ho KIM • La política coreana de seguridad energética...

(PEMEX) tiene una deuda importante, carece de recursos de inversión y no satisface la demanda interna de gas natural, insiste en monopolizar todos los sectores de la industria petrolera y de gas natural. El presidente Hugo Chávez, de Venezuela, fortaleció el control estatal de los recursos naturales mediante el decreto promulgado el 31 de marzo de 2006. PDVSA monopolizó 32 proyectos que eran llevados a cabo por empresas extranjeras. Argentina controla el precio de la energía dando prioridad al mercado nacional y grava con impuestos de $25-45 \%$ la exportación de petróleo y $20 \%$ la de gas natural, lo que desanima la inversión extranjera. Las compañías prefieren exportar gasolina que solo paga $5 \%$ de impuesto de exportación (KEXIM 2006). El presidente Evo Morales, de Bolivia, nacionalizó la industria de gas natural el 1 de mayo de 2006. Los inversionistas extranjeros fueron relegados a la calidad de subcontratistas de servicios de YPFB. Se prevé que el presidente Rafael Correa, de Ecuador, adoptará decisiones similares a las de Morales respecto del petróleo cuando asuma el mando el 15 de enero de 2007.

Esta característica del entorno de la inversión en los países latinoamericanos se refleja en las decisiones de las empresas coreanas en sus proyectos extractivos en el extranjero. Sus inversiones en Venezuela, Bolivia y Argentina ya se han visto afectadas, mientras que en Brasil y el Perú parecen estables. El estado actual de los proyectos co- reanos para el desarrollo de recursos energéticos en América Latina es el siguiente (ver Tabla 6 en la página siguiente).

Dada la situación política de la región, se amplía el papel del gobierno. De acuerdo con las orientaciones de política y las directrices antes señaladas, el gobierno coreano ha procurado cooperar en materia de energía con los países de América Latina desde principios del 2000. Al intercambiar delegaciones gubernamentales, incluso con motivo de visitas de jefes de Estado y de gobierno, el Comité de Cooperación de Recursos sostuvo reuniones con Perú para la explotación de petróleo, gas natural y cobre en julio de 2003 y noviembre de 2005; con Chile sobre cobre, en noviembre de 2004; con Brasil sobre hierro y petróleo, en mayo de 2005; con Argentina sobre cobre y petróleo, en junio de 2006; y con México sobre cobre y oro, en junio de 2006. Las delegaciones son integradas por autoridades de gobierno, funcionarios de compañías privadas y expertos en cuestiones energéticas, que examinan los detalles de la exploración y explotación de los recursos energéticos con sus contrapartes. Otro objetivo de estas misiones es incentivar a las empresas coreanas a que aumenten el monto de la inversión y faciliten la transferencia de tecnología para desarrollar recursos energéticos en ambos países, pues la distancia geográfica y la frecuente inestabilidad macroeconómica hace que América Latina sea una región poco atractiva para ellas. En las reuniones 
TABla 6

Proyectos energéticos en América Latina

\begin{tabular}{|c|c|c|c|c|c|}
\hline País & $\begin{array}{l}\text { Proyecto } \\
\text { (Proceso) }\end{array}$ & $\begin{array}{l}\text { Compañía } \\
\text { coreana }\end{array}$ & $\begin{array}{l}\text { Inversión } \\
\text { (USD\$mil) }\end{array}$ & $\begin{array}{l}\text { Reserva } \\
\text { (mil bbls) }\end{array}$ & $\begin{array}{c}\text { Producción } \\
\text { (mil bbls) }\end{array}$ \\
\hline Venezuela & Onado (producción) ${ }^{1}$ & $\begin{array}{l}\text { Korea National Oil } \\
\text { Corp (KNOC) }\end{array}$ & 33,172 & 6,994 & 229 \\
\hline \multirow{2}{*}{ Bolivia } & Palmar (producción)² & Dong Won & 7,200 & 2,700 & 0 \\
\hline & $\begin{array}{l}\text { Palmar del Oratorio } \\
\text { (exploración) }^{2}\end{array}$ & Dong Won & 36,264 & - & - \\
\hline \multirow{3}{*}{ Perú } & Perú 8 (producción) & $\begin{array}{l}\text { KNOC, Daewoo, y } \\
\text { otras. }\end{array}$ & 775,076 & 34,000 & 2,541 \\
\hline & $\begin{array}{l}\text { Perú } 88 \text { Camisea } \\
\text { (producción) }\end{array}$ & SK & 307,166 & 106,304 & 2,067 \\
\hline & Perú 56 (exploración) & SK & 15,321 & 28,336 & - \\
\hline \multirow{4}{*}{ Argentina } & Palmar Largo (producción)² & Dong Won, etc. & 42,121 & 373 & 69 \\
\hline & El Vinalar (producción) & Goleen Oil & 12,211 & 2,130 & 41 \\
\hline & Santa Victoria (exploración) & Dong Won & 15,147 & N/A & - \\
\hline & La Brea (explotación) & Petroterra & N/A & $\mathrm{N} / \mathrm{A}$ & - \\
\hline \multirow{3}{*}{ Brasil } & BMC-8 (exploración) & SK & 42,919 & $\mathrm{~N} / \mathrm{A}$ & - \\
\hline & BMC-30 (exploración) & SK & 4,947 & $\mathrm{~N} / \mathrm{A}$ & - \\
\hline & BMC-32 (exploración) & SK & 3,217 & $\mathrm{~N} / \mathrm{A}$ & - \\
\hline Total & 13 & & $1,294,761$ & 180,837 & 4,947 \\
\hline \multicolumn{2}{|c|}{ *Porcentaje del total nacional de Corea del Sur } & & $21 \%$ & $28 \%$ & $16 \%$ \\
\hline
\end{tabular}

1 Participación reducida de acuerdo con la nacionalización de 2006, de $14.1 \%$ a 5.64\%.

2 Termina en 2006, de acuerdo con la decisión de la empresa de retirarse del país.

Fuente: MOCIE, 2006b.

realizadas, las partes centran la atención en las formas de explorar y explotar diversas minas y yacimientos petrolíferos, aumentar el comercio de energía y facilitar la transferencia de tecnología.
El 25 de abril de 2006 Corea del Sur estableció el Centro de Cooperación de Recursos en América del Sur en la Embajada de Corea en la Argentina. Su función incluye el análisis de la situación de la región en materia de re- 
cursos naturales, consolidar bases de datos, estudiar proyectos de explotación y servir de canal de cooperación entre Corea del Sur y los países de América del Sur (MOCIE, 2006c).

\section{CONCLUSIONES Y CUESTIONES PENDIENTES}

La actual dependencia de Corea del Sur, economía dinámica pero de elevado uso de energía, del Medio Oriente para su abastecimiento de energía solo será sostenible cuando se garanticen la estabilidad geoestratégica de la región y el bajo precio de la energía. De lo contrario, la diversificación regional se destaca claramente como la meta de la política de seguridad energética. A mayor plazo, Corea del Sur espera aplicar su creativa diplomacia para la seguridad energética en el noreste de Asia (Calder, 2005).

América Latina, Asia central y África son regiones importantes para la política de seguridad energética de Corea del Sur. Como fuente para la importación de energía, en la actualidad América Latina prácticamente carece de importancia, pero en el último tiempo la región ya ha sido el principal destino de la inversión coreana para la explotación de los recursos energéticos. El gobierno coreano también ha aplicado una activa estrategia de colaborar con sus socios de la región para ese objetivo estableciendo comités bilaterales y continuando su política de envío de delegaciones de alto nivel e incluso visitas de Jefes de Estado.
Por otra parte, en términos de comercio América Latina ha sido una región muy importante para la economía coreana. La inversión y la asistencia para el desarrollo han incrementado desde mediados de los años noventa. En este contexto, la cooperación en materia de energía requiere un modelo saludable, y al mismo tiempo enfrenta su inherente limitación. Al procesar su política de seguridad energética en la región, habrá que considerar el llamado «efecto competitivo» y el nuevo nacionalismo de América Latina.

Primero, cabe señalar que la dinámica de las economías asiáticas que resultó en el alza de los precios de las materias primas no solo produjo un efecto complementario, sino también un efecto competitivo o negativo en los países latinoamericanos exportadores de materias primas. El primero significa un crecimiento de los ingresos de exportación, mientras que el segundo entraña una amenaza a otros sectores, particularmente sectores manufactureros que enfrentan una competencia asiática más reñida. Ejemplo claro de ello es el efecto competitivo que tiene el crecimiento económico acelerado de China en los sectores manufactureros mexicanos y brasileños. Este efecto puede variar de un país latinoamericano a otro según la importancia de las manufacturas dentro del total de sus exportaciones. Este efecto puede ser limitado o marginal en el caso de los países que exportan principalmente materias primas.

Por lo tanto, es preciso formular 
una estrategia "ganador-ganador» tanto para los países que exportan como para los que importan. Sería ideal que el país importador más competitivo en manufacturas pudiera cooperar con el país exportador en la modernización de sus industrias, mientras el segundo cooperar con asegurar las materias primas. Otro modelo sería el de cooperación entre China y Venezuela. China ofreció un crédito de 700 millones de dólares al gobierno venezolano para su utilización en programas para combatir la pobreza a cambio de tecnología para la refinación de petróleo. La mayor parte del petróleo que China explotará en Venezuela es del tipo brea, que requiere un proceso de refinación amplio y complejo, en circunstancias de que las refinerías venezolanas han sido adaptadas para tratar el petróleo crudo nacional pesado (NYT, 1 de marzo de 2005). Una de las claves de la cooperación entre Corea y América Latina radicará en la eficacia con que se formule un modelo para el intercambio de capital y tecnología, y recursos.

Segundo, la inversión extrajera directa sostenible es generalmente bienvenida en América Latina. Pero también, la creciente presencia de las empresas multinacionales en los sectores basados en recursos naturales como consecuencia de la privatización de empresas públicas no solo se ha traducido en la apropiación de parte creciente de las utilidades por las multinacionales, sino también en su transferencia al extranjero mediante las remesas de dividendos y utilidades. Además de los incentivos fiscales ofrecidos a las multinacionales, estas tenían un impacto negativo en la capacidad de los gobiernos de obtener ingresos y promover inversiones públicas. Sin embargo, ello no significa que el Estado quede totalmente excluido de los sectores extractivos. CODELCO y Petroperú son ejemplos de la presencia significativa de las empresas públicas en la minería y los sectores petroleros. Sin embargo, un trabajo de la UNCTAD sugiere que en las actuales circunstancias, América Latina debería mejorar la infraestructura física y aumentar el gasto para resolver los problemas de la alta desigualdad de ingresos de la región, el elevado índice de desempleo y los niveles de pobreza. Señala que debería procurarse aumentar sus ingresos revisando el marco regulador y las leyes que rigen la inversión tanto extranjera como interna (Gottschalk y Prates, 2006: 25-26).

Esa filosofía política y las actuales medidas de nacionalización adoptadas en Venezuela y Bolivia en 2006 han producido una imagen negativa en los círculos de inversión en América Latina. Aunque el gobierno coreano trata de estimular a las compañías privadas a que estudien la posibilidad de cooperar en la industria extractiva de América del Sur, no podrá lograrlo a menos que se disipe su imagen negativa como inestable entorno de inversión en la región. Al respecto, por el momento para Corea del Sur, Brasil, Perú, y Colombia, con su política de apertura hacia la explotación y exportación de energía, parecen socios más prometedores. 
Won-Ho KIM • La política coreana de seguridad energética...

No obstante, las empresas extractivas de recursos naturales prefieren reducir al mínimo los riesgos y gastos de información y, además, tienden a invertir en países vecinos. Mientras Sudamérica es geográficamente la zona más alejada del noreste asiático, el sudeste de Asia es la región más conocida para las empresas. Su proximidad geográfica y los nexos étnicos y culturales de dicha región refuerzan esta estrategia. Lo mismo se aplica a Asia central (principalmente Kazajstán y Uzbekistán) que recientemente ha surgido como otra alternativa. Australia y los países africanos también emergen como socios favoritos de cooperación en materia de seguridad energética. De esta manera, aunque la dotación de recursos naturales de América Latina sea un atractivo importante para las grandes empresas coreanas que buscan asegurar fuentes estables de suministro de recursos naturales, la distancia y la carencia de eslabones culturales seguirán siendo barreras por superar para lograr que aumenten las inversiones coreanas en esta región.

\section{REFERENCIAS}

BP (British Petroleum) (2006), Statistical Review of World Energy, Londres, British Petroleum.

Calder, Kent (2005), Korea's Energy Insecurities: Comparative and Regional Perspectives, Washington, D.C., Korea Economic Institute.

DOE (Department of Energy) (2006), International Energy Annual 2004, Was- hington, D.C., Department of Energy, Energy Information Administration.

IHT (2006), «South Korea practices 'resource diplomacy': New fund created for foreign deals», International Herald Tribune, 2 de octubre.

IMF (2005). World Economic Outlook, Chap. I. International Monetary Fund. Washington, D.C. abril.

Gottschalk, Ricard y Daniela Prates (2006), «East Asia's Growing Demand for Primary Commodities - Macroeconomic Challenges for Latin America», UNCTAD G-24 Discussion Paper Series No. 39, Naciones Unidas, Nueva York y Ginebra, febrero.

KEXIM (Export-Import Bank of Korea) (2006), El Estado de Recursos Energéticos y Minerales en América Latina y Dirección para la Participación de la Firmas Coreanas, Seúl: Export-Import Bank of Korea (en coreano).

MOCIE (Ministerio de Comercio, Industria, y Energía) (2006a), «Korea's Overseas Resources Development Policies», presentado en el seminario sobre cooperación entre Corea y Sudamérica en materia de reservas, Cámara coreana de Comercio e Industria, 25 de abril.

MOCIE (2006b), «Respuestas Políticas a las Medidas de Nacionalización en Sudamérica", Informativo de Prensa, 3 de mayo (en coreano).

MOCIE (2006c), «Expansión de Cooperación de Energía a América Latina», Informativo de Prensa, 27 de junio (en coreano).

MOCIE (2006d), "Comité Nacional de Energía se Reúne, Promulga Visión 2030», Informativo de Prensa, 27 de noviembre (en coreano).

MOCIE (2006e), «Inversión concentrada en explotación de recursos domésticos e internacionales», 27 de diciembre (en coreano).

NYT (2005), "China's Oil Diplomacy in Latin America", New York Times, 1 de marzo.

KEEI (Korea Energy Economics Institute) (2004a), "Reconsideration and Ways of Strengthening Energy Security», Pending Policy Issues for 2004. 
KEEI (2004b), «Measures concerning Korea's Overseas Oil Development Project», Pending Policy Issues for 2004.

Ryu, Ji-chul (2005), «Korea's Policy Measures for Energy Security», Journal of International Peace (The Seoul Peace Prize Cultural Foundation), Vol. 2, No. 1, junio, pp. 161-201. 